Applied Mathematical Sciences, Vol. 9, 2015, no. 11, 507 - 514
HIKARI Ltd, www.m-hikari.com
http://dx.doi.org/10.12988/ams.2015.411941

\title{
1-Movable Connected Dominating Sets in Graphs
}

\author{
Jocecar Lomarda ${ }^{1}$ \\ College of Teacher Education \\ Bohol Island State University-Main Campus \\ CPG North Avenue, 6300 Tagbilaran City, Bohol, Philippines \\ Sergio R. Canoy, Jr. \\ Department of Mathematics and Statistics \\ Mindanao State University-Iligan Institute of Technology \\ Tibanga Highway, 9200 Iligan City, Philippines
}

Copyright (c) 2014 Jocecar Lomarda and Sergio R. Canoy, Jr. This is an open access article distributed under the Creative Commons Attribution License, which permits unrestricted use, distribution, and reproduction in any medium, provided the original work is properly cited.

\begin{abstract}
A connected dominating set $C$ in a connected nontrivial graph $G$ is a 1-movable connected dominating set in $G$ if for every $v \in C$, either $C \backslash\{v\}$ is a connected dominating set, or there exists a vertex $u \in(V(G) \backslash C) \cap N(v)$ such that $(C \backslash\{v\}) \cup\{u\}$ is a connected dominating set of $G$. The minimum cardinality of a 1-movable connected dominating set of $G$, denoted by $\gamma_{m c}^{1}(G)$ is the 1-movable connected domination number of $G$. A 1-movable connected dominating set with cardinality $\gamma_{m c}^{1}(G)$ is called a minimum 1-movable connected dominating set or a $\gamma_{m c^{-}}^{1}$ set of $G$. In this paper, we characterize those graphs $G$ having a 1-movable connected dominating set. We also characterize the 1-movable connected dominating sets in the join of graphs and determine the corresponding 1-movable connected domination number of these graphs.
\end{abstract}

\section{Mathematics Subject Classification: 05C69}

\footnotetext{
${ }^{1}$ This research is partially funded by the Commission on Higher Education, Philippines under Faculty Development Program Phase II
} 
Keywords: connected domination, 1-movable connected domination, external and internal private neighbors, join

\section{Introduction}

Let $G=(V(G), E(G))$ be a graph with $n=|V(G)|$ and $m=|E(G)|$. For any vertex $v \in V(G)$, the open neighborhood of $v$ is the set $N_{G}(v)=N(v)=\{u \in V(G): u v \in E(G)\}$ and the closed neighborhood of $v$ is the set $N_{G}[v]=N[v]=N(v) \cup\{v\}$. If $S \subseteq V(G)$, then the open neighborhood of $S$ is the set $N_{G}(S)=N(S)=\cup_{v \in S} N_{G}(v)$ and the closed neighborhood of $S$ is the set $N_{G}[S]=N[S]=S \cup N(S)$. A set $S \subseteq V(G)$ is a dominating set of $G$ if for every $v \in V(G) \backslash S$, there exists $u \in S$ such that $u v \in E(G)$, that is, $N_{G}[S]=V(G)$. The domination number of $G$, denoted by $\gamma(G)$ is the smallest cardinality of a dominating set of $G$. A dominating set of $G$ with cardinality equal to $\gamma(G)$ is called a $\gamma$-set of $G$. Now, if $S$ is a dominating set of $G$, then a vertex $u$ is a private neighbor of $v \in S$ if $N(u) \cap S=\{v\}$. If $u \in S$, then $u$ is an internal private neighbor of $v \in S$, otherwise, $u$ is an external private neighbor of $v \in S$. The set of internal private neighbors of $v \in S$ with respect to $S$ is denoted by $i p n(v ; S)$ and the set of external private neighbors of $v \in S$ with respect to $S$ is denoted by epn $(v ; S)$.

A dominating set $S \subseteq V(G)$ is called a connected dominating set of $G$ if the subgraph $\langle S\rangle$ induced by $S$ is connected. The connected domination number of $G$, denoted by $\gamma_{c}(G)$ is the smallest cardinality of a connected dominating set of $G$. A connected dominating set $S$ of $G$ with $|S|=\gamma_{c}(G)$ is called a $\gamma_{c}$-set. A connected dominating set $C$ in $G$ is a 1-movable connected dominating set of $G$ if for every $v \in C$, either $C \backslash\{v\}$ is a connected dominating set, or there exists a vertex $u \in(V(G) \backslash C) \cap N(v)$ such that $(C \backslash\{v\}) \cup\{u\}$ is a connected dominating set of $G$. The minimum cardinality of a 1-movable connected dominating set of $G$, denoted by $\gamma_{m c}^{1}(G)$ is the 1-movable connected domination number of $G$. A 1-movable connected dominating set with cardinality $\gamma_{m c}^{1}(G)$ is called a minimum 1-movable connected dominating set or a $\gamma_{m c}^{1}$-set of $G$. Moreover, 1-movable domination and 1-movable total domination in graphs are introduced and investigated in [1], [2], and [3].

\section{Results}

Remark 2.1 Every connected dominating set contains every cut-vertex.

The next result characterizes all connected nontrivial graphs having a 1-movable connected dominating set. 
Theorem 2.2 A connected nontrivial graph $G$ has a 1-movable connected dominating set if and only if $G$ has no cut-vertices.

Proof. Suppose that $G$ has a 1-movable connected dominating set, say $S$. Suppose further that $G$ has a cut-vertex $v$. Then, by the remark 2.1, $v \in S$. Hence, $S \backslash\{v\}$ and $(S \backslash\{v\}) \cup\{u\}$, where $u \in V(G) \backslash S$ are not connected dominating sets of $\mathrm{G}$. This implies that $S$ is not a 1-movable connected dominating set, contrary to our assumption. Thus, $G$ has no cut-vertices.

Conversely, suppose that $G$ has no cut-vertices. Let $S=V(G)$. Then, clearly, $S$ is a connected dominating set. Let $v \in S$. Since $G$ has no cutvertices, $S \backslash\{v\}$ is a connected dominating set of $G$. Hence, $S$ is a 1-movable connected dominating set of $G$.

Remark 2.3 For any connected nontrivial graph $G$ without cut-vertices, $\gamma_{c}(G) \leq \gamma_{m c}^{1}(G)$.

Remark 2.4 Let $G$ be a connected nontrivial graph without cut-vertices. Then $1 \leq \gamma_{m c}^{1}(G) \leq n$, where $n=|V(G)|$, and these bounds are sharp.

To see this, consider $G_{1}=C_{4}$ and $G_{2}=K_{5}$. It can be verified that $\gamma_{m c}^{1}\left(G_{1}\right)=\gamma_{m c}^{1}\left(C_{4}\right)=4$ and $\gamma_{m c}^{1}\left(G_{2}\right)=\gamma_{m c}^{1}\left(K_{5}\right)=1$.

The next result says that all nontrivial complete graphs attain the lower bound of the inequality in Remark 2.4.

Lemma $2.5 \gamma_{m c}^{1}\left(K_{n}\right)=1$ for all $n \geq 2$.

Proof. Choose any $x \in V\left(K_{n}\right)$ and let $S=\{x\}$. Then $S$ is a connected dominating set of $K_{n}$. If $y \in V\left(K_{n}\right) \backslash\{x\}$, then $(S \backslash\{x\}) \cup\{y\}=\{y\}$ is a connected dominating set of $G$. Thus, $S$ is a 1-movable connected dominating set of $K_{n}$. Therefore, by Remark $2.4, \gamma_{m c}^{1}\left(K_{n}\right)=1$.

Theorem 2.6 Let $G$ be a connected nontrivial graph without cut-vertices. Then $\gamma_{m c}^{1}(G)=1$ if and only if $G=K_{2}$ or $G \cong K_{2}+H$ for some graph $H$.

Proof. Suppose that $\gamma_{m c}^{1}(G)=1$. If $|V(G)|=2$, then $G=K_{2}$. Suppose that $|V(G)|>2$. Then $G$ has a $\gamma_{m c}^{1}$-set say, $S=\{x\}$ for some $x \in V(G)$. Since $x$ dominates $G$, it follows that $V(G) \backslash\{x\} \subseteq N(x)$. Since $\mathrm{S}$ is a 1movable connected dominating set of $G$, there exists $y \in(V(G) \backslash S) \cap N(x)$ such that $(S \backslash\{x\}) \cup\{y\}=\{y\}$ is a connected dominating set of $G$. Hence, $V(G) \backslash\{y\} \subseteq N(y)$. Thus, $x y \in E(G)$. Let $H=\langle V(G) \backslash\{x, y\}\rangle$. Then, $G=\langle\{x, y\}\rangle+H \cong K_{2}+H$.

Conversely, if $G=K_{2}$, then by Lemma 2.5, $\gamma_{m c}^{1}(G)=\gamma_{m c}^{1}\left(K_{2}\right)=1$. Suppose that $G \cong K_{2}+H$ for some graph $H$. Let $V\left(K_{2}\right)=\{a, b\}$ and set $S=\{a\}$. Then $S$ is a connected dominating set of $G$ and $S \backslash\{a\} \cup\{b\}=\{b\}$ is a connected dominating set of $G$. Thus $S$ is a $\gamma_{m c}^{1}$-set of $G$. Thus, $\gamma_{m c}^{1}(G)=|S|=1$. 
Theorem 2.7 Let $G$ be a connected graph of order $n \geq 3$ having no cut-vertices. Then $\gamma_{m c}^{1}(G)=2$ if and only if the following conditions hold:

(i) $G \supsetneqq K_{2}+H$ for any graph $H$; and

(ii) there exist adjacent vertices $x$ and $y$ that dominate $G$ such that

(a) epn $(x ;\{x, y\}) \subseteq N_{G}(z)$ for some $z \in N_{G}(x) \cap N_{G}(y)$ and

(b) epn $(y ;\{x, y\}) \subseteq N_{G}(w)$ for some $w \in N_{G}(x) \cap N_{G}(y)$.

Proof. Suppose that $\gamma_{m c}^{1}(G)=2$. Then by Theorem 2.6, $(i)$ holds. Let $S=\{x, y\}$ be a $\gamma_{m c}^{1}$-set of $G$. Since $S$ is a connected dominating set of $G$, $x y \in E(G)$. Also, since $S$ is a 1-movable connected dominating set of $G$, there exists $z \in N_{G}(x)$ such that $(S \backslash\{x\}) \cup\{z\}=\{y, z\}$ is a connected dominating set of $G$. Hence, $y z \in E(G)$, that is, $z \in N_{G}(x) \cap N_{G}(y)$. Let $v \in \operatorname{epn}(x, S)$. Since $v y \notin E(G)$ and $\{z, y\}$ is a dominating set of $G$, it follows that $v \in N_{G}(z)$. Since $v$ was arbitrarily chosen, epn $(x ;\{x, y\}) \subseteq N_{G}(z)$. Similarly, (b) holds.Thus, (ii) holds.

Conversely, suppose that $(i)$ and $(i i)$ holds. Then, by Theorem 2.6, $\gamma_{m c}^{1}(G) \geq 2$. Let $S=\{x, y\}$ where $x$ and $y$ satisfy $(i i)$. Then $S$ is a connected dominating set of $G$. Moreover, by $(a),(S \backslash\{x\}) \cup\{z\}=\{y, z\}$ is a connected dominating set of $G$. Similarly, by $(b),(S \backslash\{y\}) \cup\{w\}=\{x, w\}$ is a connected dominating set of $G$. Thus, $S$ is a 1-movable connected dominating set of $G$ and so a $\gamma_{m c}^{1}$-set of $G$. Thus, $\gamma_{m c}^{1}(G)=|S|=2$.

The next result characterizes the concept of 1-movable connected dominating set in terms of the concept of private neighbors.

Theorem 2.8 Let $G$ be a connected graph without cut-vertices. A subset $S$ of $V(G)$ is a 1-movable connected dominating set of $G$ if and only if $S$ is a connected dominating set of $G$ and for each $v \in S$, either $\operatorname{epn}(v ; S)=\operatorname{ipn}(v ; S)=\varnothing$ or there exists $u \in(V(G) \backslash S) \cap N(v)$ such that $\operatorname{epn}(v ; S) \cup \operatorname{ipn}(v ; S) \subseteq N[u]$.

Proof. Suppose that $S$ is a 1-movable connected dominating set of $G$. Then $S$ is a connected dominating set of $G$. Let $v \in S$. If $S \backslash\{v\}$ is a connected dominating set of $G$, then every vertex $w$ in $(V(G) \backslash S) \cap N(v)$ is adjacent to some vertex in $S \backslash\{v\}$. This implies that $\operatorname{epn}(v ; S)=\varnothing$. Also since $\langle S \backslash\{v\}\rangle$ is connected, $\operatorname{ipn}(v ; S)=\varnothing$. Suppose that $S \backslash\{v\}$ is not a connected dominating set of $G$. Then, by assumption, there exists a vertex $u \in(V(G) \backslash S) \cap N(v)$ such that $S_{v}=(S \backslash\{v\}) \cup\{u\}$ is a connected dominating set of $G$. Let $z \in \operatorname{epn}(v ; S)$. Then $z \in N[u]$ since $S_{v}$ is a dominating set of $G$. Thus, $\operatorname{epn}(v ; S) \subseteq N[u]$. Also, if $y \in \operatorname{ipn}(v ; S)$, then $y \in N(u)$ since $\left\langle S_{v}\right\rangle$ is connected. Thus, $\operatorname{epn}(v ; S) \cup i p n(v ; S) \subseteq N[u]$. 
For the converse, suppose that $S$ is a connected dominating set satisfying the given condition. Let $v \in S$. If $\operatorname{epn}(v ; S)=i p n(v ; S)=\varnothing$, then $S \backslash\{v\}$ is a connected dominating set of $G$. Suppose that there exists $u \in(V(G) \backslash S) \cap N(v)$ such that $\operatorname{epn}(v ; S) \cup i p n(v ; S) \subseteq N[u]$. Set $S_{v}=(S \backslash\{v\}) \cup\{u\}$ and let $x \in V(G) \backslash S_{v}$. If $x=v$ or $x \in \operatorname{epn}(v ; S)$, then $x u \in E(G)$. If $x \notin\{v\} \cup \operatorname{epn}(v ; S)$, then $x y \in E(G)$ for some $y \in S \backslash\{v\}$ since $S$ is a dominating set of $G$. Moreover, since $\operatorname{ipn}(v ; S) \subseteq N(u),\left\langle S_{v}\right\rangle$ is connected. Thus, $S_{v}$ is a connected dominating set of $G$. This shows that $S$ is a 1 -movable connected dominating set of $G$.

The next result characterizes the 1-movable connected dominating sets in the join of two connected nontrivial graphs.

Theorem 2.9 Let $G$ and $H$ be connected nontrivial graphs. Then $S \subseteq V(G+H)$ is a 1-movable connected dominating set of $G+H$ if and only if one of the following statements holds:

(i) $S$ is a connected dominating set of $G$ such that if $|S|=1$, then either $S$ is a 1-movable connected dominating set of $G$ or there exists $u \in V(H)$ such that $\{u\}$ is a (connected) dominating set in $H$.

(ii) $S$ is a connected dominating set of $H$ such that if $|S|=1$, then either $S$ is a 1-movable connected dominating set of $H$ or there exists $v \in V(G)$ such that $\{v\}$ is a (connected) dominating set in $G$.

(iii) $S \cap V(G) \neq \varnothing$ and $S \cap V(H) \neq \varnothing$.

Proof. Let $S \subseteq V(G+H)$ be a 1-movable connected dominating set of $G+H$. If $S \cap V(G) \neq \varnothing$ and $S \cap V(H) \neq \varnothing$, then (iii) holds. Suppose that $S \cap V(G)=\varnothing$ or $S \cap V(H)=\varnothing$. Then $S \subseteq V(G)$ or $S \subseteq V(H)$. Suppose that $S \subseteq V(G)$. Since $S$ is a connected dominating set of $G+H$, it follows that $S$ is also a connected dominating set of $G$. Suppose that $|S|=1$, say $S=\{v\}$ for some $v \in V(G)$. Since $S$ is a 1-movable connected dominating set of $G+H$, there exists $u \in(V(G+H) \backslash S) \cap N(v)$ such that $(S \backslash\{v\}) \cup\{u\}=\{u\}$ is a connected dominating set of $G+H$. If $u \in V(G)$, then $(S \backslash\{v\}) \cup\{u\}=\{u\}$ is a connected dominating set of $G$. Hence, $S$ is a 1-movable connected dominating set of $G$. If $u \in V(H)$, then $(S \backslash\{v\}) \cup\{u\}=\{u\}$ is a connected dominating set of $H$. Thus, $(i)$ holds. Similarly, if $S \subseteq V(H)$, then $(i i)$ holds.

For the converse, suppose that $(i)$ holds. Then, by definition of $G+H, S$ is a connected dominating set of $G+H$. Suppose that $|S| \geq 2$. Let $v \in S$ and choose any $u \in V(H)$. Then $(S \backslash\{v\}) \cup\{u\}$ is a connected dominating set of $G+H$. Since $v$ is arbitrary, $S$ is a 1 -movable connected dominating set of $G+H$. Suppose that $|S|=1$. Then $S=\{v\}$ for some $v \in V(G)$. Suppose $S$ is a 1-movable connected dominating set of $G$. Then there exists 
$u \in(V(G) \backslash S) \cap N(v)$ such that $(S \backslash\{v\}) \cup\{u\}=\{u\}$ is a connected dominating set of $G$ (and hence of $G+H)$. If $S_{1}=\{w\}$ is a $\gamma_{c}$-set for some $w \in V(H)$, then $w \in V(H) \cap N(v)$ and $(S \backslash\{v\}) \cup\{w\}=\{w\}$ is a connected dominating set of $H$ (hence of $G+H$ ). So in either case, $S$ is a 1-movable connected dominating set of $G+H$. Similarly, if $(i i)$ holds, then $S$ is a 1-movable connected dominating set of $G+H$. Suppose (iii) holds. Then clearly, $S$ is a connected dominating set of $G+H$. Let $S_{1}=S \cap V(G) \neq \varnothing$ and $S_{2}=S \cap V(H) \neq \varnothing$. Then $S=S_{1} \cup S_{2}$. Let $v \in S$. Suppose that $v \in S_{1}$. If $\left|S_{1}\right|=1$, then there exists $u \in\left(V(G) \backslash S_{1}\right) \cap N(v)$ (since $G$ is a nontrivial connected graph) such that $(S \backslash\{v\}) \cup\{u\}$ is a connected dominating set of $G+H$. If $\left|S_{1}\right| \geq 2$, then $S_{1} \backslash\{v\} \neq \varnothing$. Hence, in this case, it follows that $S \backslash\{v\}$ is a connected dominating set. Similar arguments can be used to come up with the desired property of $S$ if $v \in S_{2}$. Therefore, $S$ is a 1-movable connected dominating set of $G+H$.

Corollary 2.10 Let $G$ and $H$ be connected nontrivial graphs. Then

$$
\gamma_{m c}^{1}(G+H)=\left\{\begin{array}{l}
1, \text { if } \gamma_{c}(G)=1=\gamma_{c}(H) \text { or } \gamma_{m c}^{1}(G)=1 \text { or } \gamma_{m c}^{1}(H)=1 \\
2, \text { otherwise. }
\end{array}\right.
$$

Theorem 2.11 Let $H$ be a connected nontrivial graph. Then $S \subseteq V\left(K_{1}+H\right)$ is a 1-movable connected dominating set of $K_{1}+H$ if and only if one of the following statements holds:

(i) $S=V\left(K_{1}\right)$ and there exists $u \in V(H)$ such that $\{u\}$ is a (connected) dominating set in $H$.

(ii) $S=V\left(K_{1}\right) \cup S_{1}$, where $\varnothing \neq S_{1} \subseteq V(H)$ and either

(a) $S_{1}$ is a connected dominating set of $H$ or

(b) $S_{1} \cup\{c\}$ is a connected dominating set of $H$ for some $c \in V(H) \backslash S_{1}$.

(iii) $S$ is a connected dominating set of $H$.

\section{Proof.}

Let $V\left(K_{1}\right)=\{z\}$. Suppose that $S$ is a 1-movable connected dominating set of $K_{1}+H$. Consider the following cases:

Case 1: $z \in S$

Suppose that $S=\{z\}$. Since $S$ is a 1-movable connected dominating set of $K_{1}+H$, there exists $u \in V(H) \cap N(z)$ such that $(S \backslash\{z\}) \cup\{u\}=\{u\}$ is a connected dominating set of $G+H$ (and hence of $H$ ). Thus, statement $(i)$ holds. Next, suppose that $S=\{z\} \cup S_{1}$ where $\varnothing \neq S_{1} \subseteq V(H)$. Since $S$ is a 1movable connected dominating set of $K_{1}+H$, either $S \backslash\{z\}=S_{1}$ is a connected 
dominating set in $K_{1}+H$ (also in $H$ ) or there exists $c \in V(H) \backslash S_{1}$ such that $S_{1} \cup\{c\}$ is a connected dominating set of $K_{1}+H$ (also in $H$ ). Therefore statement $(i i)$ holds.

Case 2: $z \notin S$

If $z \notin S$, then $S \subseteq V(H)$. Since $S$ is a connected dominating set of $K_{1}+H$, $S$ is also a connected dominating set of $H$. Hence, statement (iii) holds.

For the converse, suppose that $(i)$ holds. Then $S$ is a connected dominating set of $K_{1}+H$ and $H$ has a $\gamma_{c}$-set say $S_{1}=\{w\}$ for some $w \in V(H)$. Thus, $(S \backslash\{z\}) \cup\{w\}=\{w\}$ is a connected dominating set of $K_{1}+H$. Hence, $S$ is a 1-movable connected dominating set of $K_{1}+H$. Suppose that $(i i)$ holds. Then $S$ is a connected dominating set of $K_{1}+H$. Let $v \in S$. Suppose that $v=z$. If $(a)$ holds, then $S \backslash\{v\}=S_{1}$ is a connected dominating set of $H$ (and hence of $\left.K_{1}+H\right)$. If $(b)$ holds, then $(S \backslash\{v\}) \cup\{c\}=S_{1} \cup\{c\}$ is a connected dominating set of $H$ (and hence of $K_{1}+H$ ). Next, suppose that $v \in S_{1}$. Since $z \in S \backslash\{v\}, S \backslash\{v\}$ is a connected dominating set of $K_{1}+H$. Hence, in either case, $S$ is a 1-movable connected dominating set of $K_{1}+H$. Finally, suppose that (iii) holds. Then clearly, $S$ is a connected dominating set of $K_{1}+H$. Let $v \in S$. Then $S \backslash\{v\}) \cup\{z\}$ is a connected dominating set of $K_{1}+H$. Hence, $S$ is a 1-movable connected dominating set of $K_{1}+H$.

Corollary 2.12 Let $H$ be a connected graph of order $n \geq 2$. Then

$$
\gamma_{m c}^{1}\left(K_{1}+H\right)=\gamma_{c}(H)
$$

Theorem 2.13 Let $m \geq 2$ and $n \geq 2$ be positive integers. Then $S \subseteq V\left(K_{m, n}\right)$ is a 1-movable connected dominating set of $K_{m, n}=\overline{K_{m}}+\overline{K_{n}}$ if and only if $\left|S \cap V\left(\overline{K_{m}}\right)\right| \geq 2$ and $\left|S \cap V\left(\overline{K_{n}}\right)\right| \geq 2$.

Proof. Let $S_{1}=S \cap V\left(\overline{K_{m}}\right)$ and $S_{2}=S \cap V\left(\overline{K_{n}}\right)$. Suppose that $S$ is a 1-movable connected dominating set of $K_{m, n}$. Since $\langle S\rangle$ is connected, $S_{1} \neq \varnothing$ and $S_{2} \neq \varnothing$. Suppose that $\left|S_{1}\right|=1$, say $S_{1}=\{v\}$. Then $\langle S \backslash\{v\}\rangle=\left\langle S_{2}\right\rangle$ is not connected. Also, $\langle(S \cup\{v\}) \cup\{x\}\rangle=\left\langle S_{2} \backslash\{x\}\right\rangle$ is not connected for all $x \in V(H) \backslash S_{2}$. Thus, $S$ is not a 1-movable connected dominating set of $K_{m, n}$, contrary to our assumption. Therefore, $\left|S_{1}\right| \geq 2$. Similarly, $\left|S_{2}\right| \geq 2$.

Conversely, suppose that $\left|S \cap V\left(\overline{K_{m}}\right)\right| \geq 2$ and $\left|S \cap V\left(\overline{K_{n}}\right)\right| \geq 2$. Then $S=S_{1} \cup S_{2}$ is a connected dominating set of $K_{m, n}$. Let $v \in S$. If $v \in S_{1}$, then $S_{1} \backslash\{v\} \neq \varnothing$. Hence, $S \backslash\{v\}$ is a connected dominating set of $K_{m, n}$. Also, if $v \in S_{2}$, then $S_{2} \backslash\{v\} \neq \varnothing$. Hence, $S \backslash\{v\}$ is a connected dominating set of $K_{m, n}$. Therefore $S$ is a 1-movable connected dominating set of $K_{m, n}$.

Corollary 2.14 Let $m \geq 2$ and $n \geq 2$ be positive integers. Then

$$
\gamma_{m c}^{1}\left(K_{m, n}\right)=4
$$




\section{References}

[1] J. Blair, R. Gera and S. Horton. Movable dominating sensor sets in networks. Journal of Combinatorial Mathematics and Combinatorial Computing, 77 (2011), 103-123.

[2] R. G. Hinampas, Jr., and S. R. Canoy. 1-movable domination in graphs. Applied Mathematical Sciences, 8(2014), no. 172, 8565-8571. http://dx.doi.org/10.12988/ams.2014.410859

[3] J. Lomarda, and S. R. Canoy. 1-movable total dominating sets in graphs. International Journal of Mathematical Analysis, 8(2014), no. 55, 2703-2709. http://dx.doi.org/10.12988/ijma.2014.410326

Received: November 17, 2014; Published: January 7, 2015 